\title{
Apuntes críticos sobre la implementación jurídica del principio cooperativo de "adhesión voluntaria y abierta» en Cuba
}

Critic's comments about the legal implementation of the cooperative principle of «voluntary and open membership» in Cuba

Orestes Rodríguez Musa ${ }^{1}$

Universidad de Pinar del Río (Cuba)

Orisel Hernández Aguilar²

Universidad de Pinar del Río (Cuba)

Sumario: I. Introducción. II. El principio de «adhesión voluntaria y abierta»: principales formas de manifestación. II.1. Admisión del socio en la cooperativa. II.2. Cese de la condición de socio en la cooperativa. III. El principio de "adhesión voluntaria y abierta»: estado de su implementación jurídica en Cuba. III.1. Reconocimiento del principio y su materialización. III.2. Admisión de socios. III.3. Salida de socios. IV. Conclusiones. V. Bibliografía. VI. Legislación.

Summary: I. Introduction. II. The principle of «voluntary and open membership»: principal forms of manifestation. II.1. The admission of the member in the cooperative. II.2. The cease of the condition of member in the cooperative. III The principle of «voluntary and open membership»: state of its legal implementation in Cuba. III.1. Recognition of the principle and it materialization. III.2. The admission of the member. III. 3. The leave of a member. IV. Conclusion. V. Bibliography. VI. Legislation.

Resumen: El presente trabajo valora la implementación jurídico-práctica del principio de «adhesión voluntaria y abierta» en Cuba, prestando especial

1 Doctor en Ciencias Jurídicas, Profesor de Derecho Constitucional y de Introducción al Derecho Cooperativo, Departamento de Derecho, Facultad de Ciencias Sociales y Humanísticas, Universidad de Pinar del Río, Cuba. E-mail: musa@upr.edu.cu

2 Doctora en Ciencias Jurídicas, Profesora de Historia General del Estado y el Derecho y Derecho Romano, Departamento de Derecho, Facultad de Ciencias Sociales y Humanísticas, Universidad de Pinar del Río, Cuba. E-mail: oriselha@upr.edu.cu 
atención a sus efectos para los socios de la cooperativa o para aquellos que aspiren a esta condición. Para ello —en un primer momento- se precisan algunos de los requisitos comúnmente exigidos para la ordenación de los efectos de este principio. Establecido este punto de referencia, seguidamente se analizan las normas cubanas respecto al tema, apuntando los aciertos y desaciertos que deben ser considerados para el perfeccionamiento de la ordenación legal de esta materia. Cuba

Palabras clave: asociación voluntaria; puerta abierta; cooperativas en

Abstract: The present work values the legal implementation of the principle of «voluntary and open membership» in Cuba, paying special attention to its effects for the members of the cooperative or for those that aspire to this condition. For it -in a first moment- they are necessary some of the requirements commonly demanded for the ordination of the effects of this principle. Established this reference point, subsequently the Cuban norms are analysed regarding the topic, the successes and mistakes that should be considered for the improvement of the legal ordination of this matter pointing.

Keywords: voluntary membership; open door; cooperative in Cuba 


\section{Introducción}

Las cooperativas, en tanto son organizaciones voluntarias, se conciben como entidades abiertas a todas las personas capaces de utilizar sus servicios y dispuestas a aceptar las responsabilidades de asociarse, sin discriminaciones raciales, políticas, religiosas, sociales, ni de género.

En esto estriba, esencialmente, el principio de «adhesión voluntaria y abierta», al reafirmar la importancia de que las personas elijan, por voluntad propia, asumir un compromiso cooperativo. No se puede forzar a las personas a hacerse cooperativistas, sino que se les debe dar la oportunidad de estudiar y comprender los valores por los que ellas existen y las ventajas socioeconómicas que potencian. De ello depende - en gran medida - la eficacia de cualquier proceso de cooperativización.

Además de "voluntaria», la asociación a la cooperativa es «abierta», a diferencia de la empresa capitalista que es cerrada. La compra de una acción en las sociedades anónimas - por ejemplo- es sobre todo una alternativa financiera, más que una verdadera incorporación social, y para materializarla debe hacerse una oferta suficientemente atractiva a quienes detentan la mayoría del paquete accionario. En la cooperativa, por el contrario, el principio que ahora nos ocupa, también llamado de «puerta abierta», nos muestra la esencia humanista del cooperativismo: las condiciones para la incorporación a la cooperativa deben ser mínimas e imprescindibles, estando determinadas - sobre todo- por la naturaleza misma de las operaciones pretendidas y nunca por una sobrevaloración del capital, lo cual reafirma también el básico compromiso de las cooperativas de reconocer la dignidad de todos los individuos, sin discriminación alguna (BASAÑES 1979, 16-18).

Por otra parte, este principio recuerda a los asociados que, junto a los derechos que por la condición de socios les corresponde, tienen deberes para con sus cooperativas, los cuales incluyen sobre todo la utilización de los mecanismos de dirección y control colectivo sobre la empresa y el aporte de capital cuando este corresponda. Se trata de un conjunto de exigencias que demandan se les dedique especial atención, pero que podrían redundar en importantes beneficios tanto para los socios como para la asociación.

Regular a la cooperativa en las legislaciones internas sobre la base de esta premisa, significa ponderar principios jurídicos tan importantes como la autonomía de la voluntad y la igualdad. El primero exigirá la primacía de normas dispositivas al tutelar la libertad de asociarse en cooperativas, sin limitaciones ni imposiciones; el segundo, además de 
concebirse formalmente en el libre acceso a la forma asociativa (igualdad ante la ley), también en esta asociación puede encontrará realización efectiva (igualdad material) (RODRíGUEz MUSA 2017, 46).

En consecuencia, es posible afirmar que este principio transversaliza el desenvolvimiento del socio en la cooperativa $y$, por ende, "es indiscutiblemente el más poderoso - pero a menudo el más infravalorado- de todos los principios cooperativos» (ACl 1995). Por ello, no sorprende el alto nivel de consagración con que cuenta en las normas de la materia.

En este trabajo interesa valorar la implementación jurídico-práctica del principio de «adhesión voluntaria y abierta» en Cuba, prestando especial atención a sus efectos para los socios de la cooperativa o para aquellos que aspiren a esta condición. Para ello -en un primer momento- se precisan desde la doctrina del Derecho Cooperativo algunos de los aspectos más notables del contenido de este principio. Establecido este punto de referencia, seguidamente se analizan las normas cubanas respecto al tema, apuntando los aciertos y desaciertos que deben ser considerados para el perfeccionamiento de la ordenación legal de esta materia.

\section{El principio de uadhesión voluntaria y abierta»: principales formas de manifestación}

Dado el alcance que posee dentro de la dinámica de la cooperativa la «adhesión voluntaria y abierta», este principio manifiesta sus efectos tanto en el proceso de ingreso del socio a la cooperativa, como en los casos en que cesa esta condición.

\section{II.1. Admisión del socio en la cooperativa}

La admisión, o más bien, el proceso que conduce a ella, es la primera de las etapas de la relación del individuo con la cooperativa en condición de participante de la actividad de esta. En consecuencia, el socio potencial adquirirá determinados derechos y obligaciones respecto a ella (García-Jiménez 2001, 35; GarCíA Müller 2016, 265).

De partida, debe primar en el proceso el respeto a la voluntad del sujeto a incorporarse, así como la posibilidad de que el ingreso esté disponible para todos aquellos que así lo soliciten, en tanto no debe existir ningún indicio de trato preferente o discriminatorio. No obstante, en este último aspecto se han venido abriendo paso, tanto en las leyes 
como en los estatutos, previsiones de situaciones que limitan el acceso a la condición de socio.

Si bien la ley no puede prever todos los supuestos de admisión o de rechazo de los aspirantes a miembros, sí se ha de requerir a las cooperativas para que en sus estatutos concreten estos requisitos previo al inicio del proceso de admisión (Romero, 2001), a fin de prevenir vulneraciones a la naturaleza del principio. Entre las restricciones comúnmente aceptadas para rechazar la admisión de socios en las cooperativas figuran:

\section{a. LIMITAR EL INGRESO DE MENORES DE EDAD.}

Sobre este particular HeNRY $(2013,81)$ señala que «la posibilidad de que los menores se afilien por sí mismos a una cooperativa requiere un estudio cuidadoso de sus implicaciones en términos de responsabilidad, de las obligaciones, del derecho a voto y de elegibilidad para los cargos de responsabilidad»; aunque advierte que pueden hacerse excepciones para las cooperativas escolares y estudiantiles.

Sobre este particular, la Ley Marco para las Cooperativas de América Latina (ACI-AmÉRICAS 2008, artículo 21) no se compromete a recomendar una edad en específico, sino que reserva esta decisión a los legisladores nacionales, en tanto se trata de un aspecto que debe ser coherente con cada ordenamiento jurídico y con cada contexto.

\section{b. No SUPERAR LA CANTIDAD MÍNIMA O MÁXIMA DE ASOCIADOS}

La fijación de estas cantidades, tanto mínimas como máximas, puede depender de la función de la cooperativa o de otros criterios que los asociados consideran relevantes, siempre en función de ajustar el número de socios a la viabilidad y eficiencia en el desarrollo de la cooperativa.

A tono con ello se reconoce que «la única restricción posible será la derivada de las propias condiciones del objeto social, ya que no podría obligarse a la cooperativa a seguir incorporando socios cuando su capacidad de prestación de servicios estuviera colmada» (ACI-AMÉRICAS 2008, 11), de no ser así no existe justificación para imponer cortapisas al crecimiento de estas entidades.

\section{NO ADMITIR INTERESES EN CONFLICTO O DESLEALTAD CON LA COOPERATIVA}

Esta exigencia puede concretar en que se soliciten determinadas condiciones morales de los aspirantes, siempre que puedan ser obje- 
tivamente apreciadas y que tengan causa justificada por su relevancia (Orgaz 2006). Además, existen prohibiciones de doble afiliación, sobre todo si la segunda le produce algún daño a la entidad de primera afiliación (GARCíA MülleR 2016, 259).

\section{d. TENER CuALIDADES PROFESIONALES A FINES AL OBJETO SOCIAL DE LA COOPERATIVA}

Se trata de la existencia de correspondencia entre los requisitos subjetivos de los socios con el objeto social de la cooperativa (PAOLucCl, 1999), de forma tal que de acuerdo a sus características, pueda vincularse a la actividad propia de la cooperativa (GARCía Müller 2016, 256). Dicho así, este requisito puede acarrear — con relativa facilidad- arbitrariedades y/o limitaciones ${ }^{3}$ a la sustancia del principio en cuestión.

Para ofrecer mayor seguridad en este sentido, es pertinente dotar el procedimiento de admisión de algunos términos que le impregnen transparencia, tanto para los socios como para los solicitantes de esta condición.

En tal sentido, resulta recomendable que las solicitudes de adhesión estén fechadas y confirmadas mediante un documento que acredite su recepción; que la admisión o no del solicitante sea resuelta por la Asamblea General; y que los términos para la adopción de la decisión estén debidamente fijados, así como los efectos que se le atribuyen al silencio. De igual forma, es necesario que los rechazos sean fundamentados por escrito y notificados de inmediato al solicitante; y que el solicitante cuente con el derecho a apelar frente a un tribunal de justicia (HENRŸ 2013, 83).

3 En tanto serán recurrentes en este trabajo los término de «límites» y «limitaciones», vale desde un inicio advertir que, a efectos de este estudio, se entenderá por "limitaciones jurídicas» aquellas restricciones y falencias presentes en la norma o derivadas de su interpretación/aplicación, que pueden incidir en que las instituciones no se desarrollen conforme a su naturaleza; diferentes a los necesarios «límites» que el ordenamiento jurídico impone para contribuir al normal desarrollo de las relaciones sociales. Aludiendo concretamente a los derechos fundamentales, es clarificador en este sentido NogueIRA AlCALÁ cuando explica que debe entenderse por «límite de un derecho la frontera entre lo que algo es y lo que no es... parte de la estructura del derecho y considera todos los demás derechos y bienes constitucionalmente protegidos... presupone la existencia de un contenido constitucionalmente protegido... [Por su parte] El concepto de limitación de un derecho... significa la imposición de restricciones... en la medida que el acto de limitar un derecho no puede llegar al punto de desnaturalizarlo, transformándolo en otro, haciendo imposible su reconocimiento y ejercicio... La limitación, en cuanto restricción del derecho, es un acto que procede desde fuera e implica alterar su condición natural» (NogueIRA AlCALÁ 2005, 19). 


\section{2 Cese de la condición de socio en la cooperativa}

Como bien apunta BorjabAd BelLdo $(2013,48)$, «dentro de la libre adhesión ha de comprenderse la permanencia en la vinculación societaria desde el momento en que se produjo la adhesión y ello ha de entenderse desde el punto de vista de la protección de los intereses del socio, unas veces, y de la cooperativa, otras». Por ello, tanto la legislación como los estatutos cooperativos han de regular, para bien de ambas partes del vínculo jurídico-cooperativo, los supuestos y los efectos de la desvinculación societaria.

\section{a. SALIDA VOLUNTARIA}

El derecho del socio a salir de la cooperativa debe estar garantizado por la ley y/o los estatutos en términos que proteja su voluntad; pero también ha de armonizarse con la subsistencia de la cooperativa.

Así pues, aunque al asociado que renuncia o se retira no se le puede denegar su solicitud y debe reembolsársele sus aportes sociales, en estos casos es posible establecer ciertas condiciones favorables a la cooperativa. Entre estas condiciones se reconocen la de cumplir con un período mínimo de vinculación; saldar los principales compromisos financieros asumidos; aguardar por el reembolso un plazo de tiempo determinado y razonable, cuando una devolución de este al socio por la cooperativa pudiera afectar seriamente el funcionamiento de esta, pagándose un interés limitado sobre el importe a ser reembolsado (HENRY 2013, 83-84).

\section{b. SALIDA INVOLUNTARIA}

En correspondencia con la apertura de la entrada a la cooperativa, los supuestos de exclusión o suspensión de sus miembros han de tener carácter excepcional. Puede «producirse cuando los asociados no se retiren voluntariamente, aun cuando ya no cumplen con los requisitos para ser asociados, cuando hayan violado seriamente los estatutos o cuando su comportamiento fuera perjudicial para la cooperativa por cualquier otro motivo» (HENRŸ 2013, 84).

Dada la naturaleza involuntaria de las medidas que por estos motivos excepcionales se adopten, ha de centrarse la atención en que este procedimiento sea aún más seguro y garantista que el de admisión. Por ende, se le debe ofrecer al socio toda la información sobre el proceso en su contra, así como el derecho a ser escuchado. También debe contar con la posibilidad de acceder a los procedimien- 
tos de solución de controversias previstos en la ley o en los estatutos; ofrecérsele la posibilidad a apelar ante la Asamblea General y, como último recurso, el de acudir a un tribunal imparcial (HENRŸ 2013, 84). Todo ello siguiendo los términos para la sustanciación del proceso previstos en la ley y/o los estatutos.

\section{El principio de «adhesión voluntaria y abierta»: estado de su implementación jurídica en Cuba}

En Cuba, hasta hace solo unos pocos años, solo eran admisibles las cooperativas de carácter agropecuario, entre las que figuraban tres tipos: las Cooperativas de Producción Agropecuarias (CPA), las Cooperativas de Créditos y Servicios (CCS) y las Unidades Básicas de Producción Cooperativas (UBPC), cada una de ellas con características diferentes y normas propias. En el año 2012, con la autorización para constituir Cooperativas No Agropecuarias (CNA), se expandieron estas formas asociativas a otras actividades económicas. Sin embargo, ello ha ocasionado que la legislación cooperativa en Cuba, responde a bases contextuales y concepciones diferentes sobre la figura, existiendo en la actualidad una seria dispersión normativa en la materia.

Así pues, para conocer a cabalidad cualquier cuestión relativa al sector cooperativo en Cuba, es preciso sistematizar la información que ofrecen, entre otras, la Ley No. 95 de 2002, De las Cooperativas de Producción Agropecuaria y de Créditos y Servicios (L95/02); el Decreto-Ley No. 142 de 1993, Sobre las Unidades Básicas de Producción Cooperativa (DL142/93); el Decreto-Ley No. 305 de 2012, De las Cooperativas No Agropecuarias (DL305/12); el Acuerdo No. 5454 del Comité Ejecutivo del Consejo de Ministros, contentivo del Reglamento General de las Cooperativas de Producción Agropecuaria [Anexo 1] (RG-CPA) y del Reglamento General de las Cooperativas de Créditos y Servicios [Anexo 2] (RG-CCS); la Resolución No. 574 de 2012 del Ministerio de la Agricultura, Reglamento General de las Unidades Básicas de Producción Cooperativa (RG-UBPC); y el Decreto No. 309 de 2012, Reglamento de las Cooperativas No Agropecuarias de Primer Grado (R-CNA).

\section{III.1. Reconocimiento del principio y su materialización práctica}

En los cuerpos normativos que ordenan el desenvolvimiento de cada una de las modalidades cooperativas cubanas, se observa con fa- 
cilidad el reconocimiento de la naturaleza «voluntaria» de estas formas asociativas. No obstante, un análisis más profundo sobre estas normas jurídicas, nos lleva a dilucidar las limitaciones que su implementación jurídico-práctica impone —-sobre todo- a los socios o a los aspirantes a serlo.

Al regular de conjunto a las CPA y a las CCS, la L95/02 establece, en el mismo precepto (artículo 3, inciso a.) ${ }^{4}$, que se tomará en cuenta la voluntad de los miembros para su incorporación y, también, para su permanencia en las cooperativas. Esta fórmula es también seguida por el DL305/12 al regular a las CNA (artículo 4, inciso a.). En el caso de las UBPC, la «voluntariedad» está presente como parte del concepto que de ellas se ofrece en el artículo 1 de su Reglamento General.

Sin embargo, es pertinente resaltar que las normas cubanas que regulan las formas cooperadas de producción, reducen el reconocimiento del principio de «adhesión voluntaria y abierta» a su expresión formal de la «voluntariedad» para ingresar y permanecer en las cooperativas, descuidándose la protección del libre acceso a las cooperativas.

Lo antes dicho permite apuntar que la primera dificultad de la legislación nacional sobre el particular que nos ocupa, radica en la técnica empleada para el reconocimiento del principio, toda vez que su plasmación como principio resulta incompleta y asistémica la ordenación de su contenido.

A tono con lo anterior, otra cuestión a considerar es la materialización práctica del principio en cada una de las tipologías cooperativas, en particular en las UBPC y las CNA. Respecto a las primeras, LOPEZ LABRADA y Rodríguez Membrado $(2012,362)$ señalan que son «una forma de organización empresarial, fruto del rediseño de la propiedad estatal, que vincula en un sistema peculiar de relaciones económicas la gestión cooperativa con la dirección centralizada», puesto que no surgieron como resultado de la iniciativa consciente de sus asociados. Nótese que en ninguno de los artículos del DL142/93 — norma que les dio nacimiento- se contempla el principio de referencia.

En cuanto a las CNA debe resaltarse que un número importante de ellas son resultado de propuestas para la conversión en cooperativas de las empresas estatales ${ }^{5}$, por lo que su constitución fue resultado de una propuesta administrativa unilateral desde la entidad a la que estas unidades se subordinaban, dando cumplimiento a polí-

4 Vid. Además artículo 4 del RG-CPA y artículo 5 del RG-CCS.

5 De las primeras 498 CnoA autorizadas, 384 (77\%) son de origen estatal y solo 114 (23\%) de iniciativa privada (PIÑEIRO HARNECKER 2014, 10). 
ticas trazadas centralmente por sus respectivos Ministerios ${ }^{6}$. Por ello se ha dicho que los «socios sienten que la decisión se les impuso, que realmente no les preguntaron si querían formar una cooperativa o no, sino que más bien les «informaron» que se iba a crear...»(PINEIRO HARNECKER 2014, 13), lo cual significa una realidad generalizable a todo el país en el caso de las cooperativas inducidas desde el Estado (Vuotto 2015, 17). Cierto es que a los trabajadores de estas entidades nadie los forzó materialmente a asociarse, tuvieron la alternativa de no hacerlo; pero ante su negativa, la opción laboral era declararse disponibles ${ }^{7}$, por lo que prefirieron experimentar.

Esta falta de voluntariedad real (vs. formal) conspira contra la identidad universalmente reconocida a las cooperativas, afectando concretamente el principio que nos ocupa, lo cual es preocupante puesto que su vulneración puede acarrear consecuencias irreversibles para la estabilidad del sector y para su credibilidad social, en tanto resulta premisa esencial de su efectiva consolidación.

\subsection{Admisión de socios}

Posterior a la constitución de la cooperativa, la primera manifestación del principio de «puertas abiertas» es la presencia — como se señaló supra - del proceso de admisión de nuevos asociados. Para hacer de este un trámite apegado al espíritu de la oportunidad para todos, con base en la igualdad, es necesario que los requisitos exigibles estén previamente determinados por la ley, de conformidad con criterios que resulten justificativos. Siguiendo este criterio, se analiza a continuación su previsión en el ordenamiento jurídico cubano.

\section{a. EDAD MÍNIMA DE LOS SOCIOS}

La edad mínima que fijan las normas para incorporar socios a las CPA y las CCS es de 16 años de edad (artículo 53 del RG-CPA y artículo 55 del RG-CCS), mientras que para ingresar a las CNA se exige tener 18 años (artículo 10, apartado 1 del DL305/12). Esta última disposición asume una edad que le obstaculiza el acceso a la condición de

6 «...no se trata de personas que se han agrupado voluntariamente en función de objetivos comunes, sino del cumplimiento de orientaciones» (COMISIÓN DE Asuntos ECOnómicos de la Asamblea Provincial del Poder Popular en Pinar del Río 2016, 7).

7 Vid. Disposición Especial Primera del DL305/12 en relación con el artículo 53, inciso c) del Código de Trabajo. 
socio a personas que, según la Ley 116 de 2014, Código del Trabajo cubano, cuentan con capacidad para concertar contratos de trabajo, la cual se adquiere a los 17 años, e incluso, excepcionalmente desde los 15 años (artículo 86 del Código de Trabajo).

Cierto es que la condición de socio de la cooperativa implica deberes y responsabilidades adicionales a la simple vinculación laboral a la cooperativa, lo cual podría justificar que, probablemente, en este caso el legislador haya tomado como referente la mayoría de edad prevista en el Código Civil cubano como regla general (artículo 29, apartado 1, inciso a.). Por tanto, este pronunciamiento del R-CNA veta el acceso inmediato a la condición de socio para los trabajadores menores de 18 años, incluso para aquellos que ya tenían contratos indeterminados con la entidad que pasa a ser gestionada como CNA. En este caso "se les contratará hasta arribar a dicha edad, momento en que procederá su ingreso como socio fundador» (Disposiciones Especiales Segunda).

Pero, si fuera la pretensión de madurez física o psicológica del individuo lo que provoca la prudencia del legislador en el caso de la mayoría de edad para formar parte de una CNA, debe tomarse en cuenta que este inconveniente puede manifestarse en individuos de cualquier edad, razón por la cual el proceso de admisión prevé la posibilidad de que puedan rechazarse justificadamente a los aspirantes. En cualquier caso, téngase en cuenta que no está resultando coherente la legislación cooperativa agropecuaria con la no agropecuaria en este aspecto.

A esta incoherencia se suma el hecho de que no existe pronunciamiento legal sobre la mayoría de edad para vincularse a las UBPC. Esto podría imputarse a que estas formas cooperadas se concibieron para ser integradas por trabajadores ya activos laboralmente en las entidades estatales del sector agropecuario y forestal, de las cuales se derivaron (RG-UBPC, artículo 2), supuestos que implican presencia de capacidad laboral reconocida. No obstante, el Reglamento también dispone que "otras personas» puedan solicitar incorporarse a las UBPC, en cuyo caso surgiría la duda de si debería asumirse para la determinación de la mayoría de edad la regla del Código Civil, del Código de Trabajo o las normas que ordenan a las otras formas de cooperativas agropecuarias (CPA y CCS).

De lo antes dicho con respecto al requisito de edad mínima para admitir nuevos integrantes en las cooperativas cubanas, se hace patente que no existe una concepción uniforme para su establecimiento, así como la presencia de un vacío legal respecto a este particular en la normativa que ordena las UBPC. 


\section{b. CANTIDAD MÁXIMA Y MÍNIMA DE SOCIOS}

Solo en el R-CNA se alude a la factibilidad de fijar en los Estatutos, la cantidad mínima y máxima de socios si se entiende preciso (artículo 21). Dado que las CPA y las UBPC son, esencialmente, cooperativas de personas que se asocian a los fines de la producción agrícola (a diferencia de las CCS, que se asocian a fin de obtener esencialmente créditos y servicios estatales), resulta difícil entender la ausencia de previsión legal respecto a este particular, toda vez que su desarrollo se puede ver afectado cuando la cantidad de asociados comprometa la viabilidad de la empresa.

No obstante, sería factible interpretar el vacío legal en favor de la autonomía para la auto-regulación cooperativa, de tal suerte que los estatutos pueden contemplar cualquier previsión al respecto, pues nadie mejor que los propios socios para ello. Recuérdese que el libre acceso a la asociación y a los beneficios cooperativos, está condicionado por la sostenibilidad de la empresa.

\section{INTERESES EN CONFLICTO}

Toda vez que las normas que regulan a las CPA, a las CCS y a las UBPC (con excepción del Reglamento General para esta última) son propias de un contexto en el que las únicas entidades de su tipo existentes eran las dedicadas a las labores agrícolas, se entiende que la asociación a una de ellas es incompatible con la vinculación a otra cooperativa ${ }^{8}$. Sin embargo, la prohibición de que un socio solo pueda realizar actividades laborales lícitas no relacionadas con la cooperativa, de manera excepcional, por un tiempo determinado y previa autorización de la Asamblea General ${ }^{9}$, ante el nuevo panorama socioeconómico existente en el país resulta injustificada.

El Reglamento de las CNA, a tono con el contexto nacional que reconoce y estimula el pluriempleo, no impone limitación al respecto; aunque se establece una reserva en el artículo 21 en favor de los estatutos, en los cuales se podrán precisar los requisitos que en cada caso se acuerden para conferir el estatus de socio a los interesados.

La previsión de los supuestos que ocasionan interese en conflicto, resulta más adecuada cuando surgen de la propia cooperativa, amén de que cuente con pautas generales desde las leyes que ordenan la actividad en pos de preservar intereses o principios generales. Por tanto, es preciso actualizar los preceptos que se ocupan de este asunto.

\footnotetext{
8 Vid. L95/02, artículo 59; RG-UBPC, artículo 53.
}

9 Vid. L95/02, artículo 59; RG-UBPC, artículo 59. 


\section{d. Aptitud para el objeto social}

Para las cooperativas de carácter agropecuario ha sido regularidad exigir que las personas que se vinculen a ellas tengan experiencia en este tipo de labores. Es así que se admiten agricultores pequeños, usufructuarios de tierras, trabajadores agrícolas y apicultores.

Además, se abre la posibilidad de incorporación como cooperativistas del cónyuge, hijos y demás familiares que dependan de la economía familiar del cooperativista o del trabajador agrícola ${ }^{10}$. Esta previsión legal está en consonancia con la política agraria cubana que reconoce las singularidades de la familia en los entornos rurales y valoriza el trabajo informal de apoyo a las labores agrícolas que brindan a diario los miembros de esta familia —en especial las mujeres- que son dependientes del trabajador directo.

Personas diferentes a estas solo son admitidas en las CPA y en las UBPC, por lo que el acceso a las CCS tendría que comenzar con el establecimiento de una relación laboral previa antes de aspirar a la condición de asociado. Teniendo en cuenta lo antes apuntado, resulta llamativo que no se especifiquen particularidades para el ingreso a las UBPC de personas excluidas de los supuestos tratados, mientras que para las CPA se deja sentado que solo podrán hacerlo una vez vencido el pe-

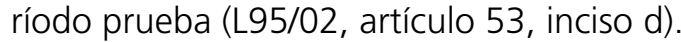

El DL305/12 declara, de manera general, que pueden ser socios de una CNA las personas naturales aptas para realizar labores productivas o de servicios de las que constituyen su actividad (artículo 10, apartado 1, inciso c). Este requisito opera como un indicador abstracto de necesaria valoración durante el proceso de solicitud de admisión por parte del órgano cooperativo encargado de decidir al respecto, reservándose a los estatutos las precisiones de rigor al respecto. En esta dirección, el R-CNA dispone expresamente, que los estatutos contendrán los requisitos que se determine exigir a los aspirantes a socios (artículo 21).

La no existencia de una formulación similar en el resto de la legislación cooperativa nacional, que flexibilice la determinación del contenido de estos requisitos en favor de los estatutos, no solo refleja la escasa autonomía (auto-regulatoria) que aún caracteriza al sector cooperativo cubano (Rodríguez Musa 2013, 142-153), sino que además dificulta lo que podría ser la custodia más eficaz del principios de «puerta abierta», es decir, aquella que emana de las propias cooperativas.

10 Vid. L95/02, artículo 53, inciso c) y artículo 55, inciso b) 


\section{e. Garantías formales del PROCESO}

La legislación cubana vigente en materia de cooperativas es bastante parca con respecto a las formalidades que debe cumplir la solicitud de un aspirante a socio, de hecho ninguna de las disposiciones relativas a las CPA, las CCS y las CNA exigen el cumplimiento de formalidades significativas en la presentación de estas solicitudes. Solo el Reglamento de las UBPC ordena que se presente un escrito de este tipo sin formalidades, firmado por los aspirantes.

Pese a esta aparente flexibilidad, el RG-UBPC también exige que este escrito sea entregado al Delegado o el Director de la Agricultura en el municipio (artículo 2), lo cual lacera la premisa de que es la Asamblea General la encargada de decidir —en última instancia - sobre la admisión del solicitante, aspecto en el que existe absoluta coincidencia en la legislación cooperativa cubana ${ }^{11}$.

Respecto a las experiencias de las CNA, ha sucedido que la incorporación o salida de los socios trasciende hasta la modificación de los estatutos $^{12}$. Esto se ha debido a que el Acta de Constitución de la Cooperativa, en la cual constan los nombres de los socios fundadores y cuyo contenido principal son los estatutos de la cooperativa, al parecer se ha confundido con estos últimos.

Sin embargo, en el R-CNA no consta ninguna exigencia para convertir los datos del socio en parte del contenido de los estatutos (artículo 21), por lo que podrían evitarse trámites engorrosos, que actualmente acarrean dificultades burocráticas de orden interno, notarial y registral con costes en tiempo y dinero (artículos 22 y 24).

En un sentido similar es oportuno referirse a la opción que significa el Reglamento de Régimen Interno (RRI), pese a que no se visualiza en la legislación cubana. Al respecto ha planteado SENENT VIDAL (2011, 68-72) que «es un acuerdo adoptado en el ejercicio de sus competencias por la Asamblea General, pero desde su aprobación es también un texto normativo interno, de rengo jurídico inferior a los estatutos... La aprobación del RRI por la Asamblea o su posterior modificación, seguirán el procedimiento general para la adopción de acuerdos sociales. En cuanto a su inscripción registral, en aquellas comunidades cuyas leyes cooperativas no contengan una mención expresa, habrá que interpretar que no es obligatoria. Por lo que respecta a las materias regulables en el RRI... los contenidos posibles son de una gran variedad y

11 Vid. RG-CPA, artículo 54; RG-CCS, artículo 56; RG-UBPC, artículo 26 y R-CNA, artículo 37, inciso e).

12 Vid. Legajos de las CNA del Registro Mercantil en Pinar del Río. 
utilidad... solo podrán excluirse aquellas disposiciones que vayan contra lo dispuesto en una norma legal o estatutaria...». Por tanto, utilizar este tipo de normativa dotaría al régimen jurídico de la persona socia de una mayor flexibilidad que si se incluyese en los estatutos sociales, ya que una eventual modificación evitaría las mayores formalidades de la modificación estatutaria, y así también se contribuiría con la eficacia del principio de «puertas abiertas».

Sobre el resto de las garantías del proceso que deben contribuir para que se respete el postulado que nos ocupa, entiéndanse los términos para la adopción de la decisión, los efectos que se atribuyen al silencio, la motivación de los rechazos y, en tales casos, el derecho a apelar ante a un tribunal de justicia, apenas se regula en la legislación cooperativa cubana. Esta situación deja en clara posición de indefensión jurídica a aquellos que son rechazados, o a los que no se les responde su solicitud, lo cual significa el quebranto del principio en cuestión.

Lo anterior ha conspirado para que se hayan dejado de aprovechar iniciativas libres y voluntarias de trabajadores por cuenta propia que se motivaron para asociarse en CNA, y que de encausárseles por esta vía hubiera servido para colectivizar su actividad económica frente al modo individual autorizado ${ }^{13}$, máxime cuando los proyectos de este tipo suelen estar bien sustentados ${ }^{14}$. Contrario a ello, se ha entendido la «masividad de propuestas desde la población» como un aspectos que afecta el éxito del experimento (TRISTÁ ARBEZú 2015). Por estos motivos, muchos se desestimulan, desisten de constituirse como CNA y se organizan como cuentapropistas, mientras que otros inician su funcionamiento sin estar constituidos formalmente (Vuotro 2015, 17), escapando de la ordenación jurídica hechos esenciales como los de la asociación voluntaria y por iniciativa propia.

13 «Existe presión desde abajo para la formación de cooperativas a partir de la asociación de trabajadores por cuenta propia, pero las mismas se han visto muy limitadas. En Pinar del Río más de 20 propuestas». Vid. Comisión de Asuntos EConómicos DE la Asamblea Provincial del Poder Popular en Pinar del Río: Estudio sobre el Desarrollo del Sector no Estatal en la Provincia y sus impactos en el nivel y calidad de vida de la población, facilitado al autor por Efraín Echevarría, Presidente de esta Comisión, p. 10.

14 Se ha apuntado por directivos nacionales que «...los proyectos espontáneos de personas naturales, o sea, que no se desprenden del sector estatal, muestran mayor dominio de las normas, del mercado y de la gestión económica. Además, están muy bien documentados jurídicamente». Vid. CASTRO, Y. \& otros: «Cooperativas no Agropecuarias en Cuba: salto con obstáculos», Periódico Granma, 8 de mayo de 2015, recuperado de http://www.cubainformacion.tv/index.php/economia/62594-cooperativas-no-agropecuarias-en-cuba-salto-con-obstaculos, el 13 de mayo de 2015. 


\section{III.3. Salida de socios}

De forma general, se admite en la legislación cooperativa cubana el cese de la condición de asociado. La salida puede darse por razones voluntarias (por ejemplo solicitud propia y jubilación) e involuntarias (por ejemplo imposición como medida disciplinaria por la Asamblea General y pérdida de los requisitos para ser socio) ${ }^{15}$. En cualquiera de estos casos, existe el derecho del socio al cobro de los anticipos pendientes, de los adeudos por los bienes aportados a la cooperativa y de las utilidades que le correspondan hasta el día en que cause baja.

\section{a. SALIDA VOLUNTARIA}

Como se ha apuntado previamente, ante los supuestos de retirada voluntaria de sus miembros, las cooperativas pueden previamente adoptar alguna medida que asegure su estabilidad, sin restringir el alcance del derecho de salida del socio.

En Cuba, no se prevén restricciones por razón de un período mínimo de asociación, ni el transcurso de un plazo de tiempo determinado y razonable, ni la retención del reembolso en beneficio del funcionamiento cooperativo; solamente se exige al socio el cumplimiento de las principales obligaciones asumidas con la cooperativa ${ }^{16}$. Por tanto, el proceso de retirada voluntaria propende más a velar por el interés individual de quien se retira que por los de aquellos que persisten en mantener el emprendimiento colectivo.

\section{b. SALIDA INVOLUNTARIA}

Contrario a lo antes dicho para las salidas voluntarias, las garantías al principio de «voluntariedad» son más reducidas durante los procesos de salida de aquellos socios que no lo han pedido.

Salvo el establecimiento de causales para este tipo de separación, en ocasiones ambiguas como es el caso de «no disfrutar de buen concepto público» (L95/02, artículo 63) y la reserva de la competencia para decidirla a la Asamblea General, máxima instancia de la cooperativa, en las normas que regulan las CPA y las CCS no figuran otras previsiones garantistas. Al respecto, resalta por negativo que los Reglamentos de

15 Vid. L95/02, artículo 63; RG-CPA, artículo 96; RG-CCS, artículo 59; RG-UBPC, artículo 65 y R-CNA, artículo 30.

16 Vid. L95/02, artículo 61; RG-UBPC, artículo 51, inciso g); R-CAN, artículo 30, inciso a). 
estas cooperativas dejan claro que contra las medidas de la Asamblea General no cabe recurso alguno en la vía judicial ${ }^{17}$.

El vigente RG-UBPC aprobado en 2012, en tanto responde a un contexto mucho más garantista (es parte del proceso de actualización del modelo socioeconómico cubano iniciado fundamentalmente tras el VI Congreso del Partido en el año 2011), supera esta limitación y exige que las medidas disciplinarias sean notificadas a los infractores mediante escrito fundado (artículo 57, apartado 2), a la vez que advierte que contra lo resuelto por la Asamblea General podrá reclamarse en la vía judicial (artículo 60).

También resultado de este contexto es el R-CNA del propio año 2012, donde se dispone que sean los estatutos de las CNA donde se establezcan las medidas disciplinarias a imponer y el procedimiento para su aplicación, cumplimiento y mecanismo de impugnación (artículo 68), lo cual significa otra buena oportunidad para la auto-regulación cooperativa. Además, se prevé la mediación como primera forma para la solución de los conflictos que surjan entre los socios y la cooperativa, y cuando esta no resulte, se podrá acudir a la vía judicial, según la naturaleza del conflicto. Sin embargo, pese al paso de avance que significan estas disposiciones con respecto al resto de la legislación cooperativa patria, cabe la duda de cuál sería el tribunal competente para conocer de estos casos donde el conflicto surge de una relación jurídica de naturaleza estrictamente cooperativa, diferente a las tradicionales.

Sobre esta base, es posible advertir que la legislación cubana que regula la materia cooperativa, no en todos los casos alcanza a asegurar que la salida involuntaria de los socios cuente con todas las garantías necesarias para contribuir a la eficacia del principio de «puerta abierta».

\section{Conclusiones}

Sobre la base de los análisis anteriores se puede arribar a las siguientes conclusiones:

1. El principio cooperativo de "adhesión voluntaria y abierta» implica la libertad de las personas para ser admitidas como socios en la cooperativa y para salir de este vínculo con garantías, cuando así lo decidan. Sin embargo, este derecho debe armoni-

17 Vid. RG-CPA, artículo 77 y RG-CCS, artículo 67. 
zarse con la subsistencia de la cooperativa, por lo que también implica límites en su configuración legal y estatutaria, principalmente durante el proceso de ingreso a la cooperativa y en los casos en que los socios cesan en esta condición por razones voluntarias o involuntarias.

2. La implementación jurídica del principio en Cuba está marcada por la dispersión normativa y por la heterogeneidad de bases contextuales en las legislaciones que regulan la materia. Entre las limitaciones que se advierten, destacan una plasmación asistémica e incompleta del contenido del principio. Además se aprecian vacíos legislativos en cuanto a los términos para la adopción de las decisiones, los efectos que se atribuyen al silencio, la exigencia de motivación de los rechazos y, en tales casos, el reconocimiento del derecho a apelar ante a un tribunal de justicia. Todo ello, unido a la escasa autonomía auto-regulatoria para custodiar el principio de "puerta abierta», ha contribuido a sus distorsiones prácticas mediante la constitución de cooperativas inducidas y el no aprovechamiento de iniciativas libres y voluntarias de trabajadores por cuenta propia motivados para asociarse.

\section{Bibliografía}

ALIANZA COOPERATIVA INTERNACIONAL (ACI). 1995. «Declaración Sobre la Identidad Cooperativa», Manchester, recuperado de http://www.elhogarobrero1905.org.ar, el 12 de diciembre de 2009.

1. (ACI-AMÉRICAS). 2008. "Ley Marco para las Cooperativas de América Latina», San José, recuperado de www.aciamericas.coop, el 18 de diciembre de 2009.

2. BASAÑES, J.C. 1979. "Teoría y realidad de la economía cooperativa». Cuaderno de Cultura Cooperativa, No. 61, Ed. Intercoop, Buenos Aires.

3. BORJABAD BELLIDO, J. V. 2013. La libre adhesión y baja voluntaria del socio en la legislación cooperativa catalana y sus consecuencias económicas. Tesis Doctoral. Universidad de Lleida.

4. CASTRO, Y. \& otros. 2015. "Cooperativas no Agropecuarias en Cuba: salto con obstáculos». Periódico Granma. 8 de mayo. Recuperado de http://www.cubainformacion.tv/index.php/economia/62594-cooperativasno-agropecuarias-en-cuba-salto-con-obstaculos, el 13 de mayo de 2015.

5. COMISIÓN DE ASUNTOS ECONÓMICOS DE LA ASAMBLEA PROVINCIAL DEL PODER POPULAR EN PINAR DEL RÍO. 2016. Estudio sobre el DesarroIlo del Sector no Estatal en la Provincia y sus impactos en el nivel y calidad de vida de la población, facilitado al autor por Efraín Echevarría, Presidente de esta Comisión. 
6. GARCÍA-JIMÉNEZ, M. 2001. Autoempleo y trabajo asociado. El trabajo en la Economía Social. Córdoba.

7. GARCÍA MÜLLER, A. 2015. Derecho Cooperativo y de la Economía Social y Solidaria. Asociación Iberoamericana de Derecho Cooperativo, Mutual y de la Economía Social y Solidaria. Mérida.

8. HENRŸ, H. 2013. Orientaciones para la legislación cooperativa (2da. Edición). OIT. Ginebra.

9. LÓPEZ LABRADA, A. y RODRíGUEZ MEMBRADO, E. 2012. "La UBPC: forma de rediseñar la propiedad estatal con gestión cooperativa». En PIÑEIRO HARNECKER, C. (compiladora): Cooperativismo y Socialismo. Una mirada desde Cuba. Ed. Caminos: 337-362. La Habana.

10. NOGUEIRA ALCALÁ, H. 2005. "Aspectos de una Teoría de los Derechos Fundamentales: La Delimitación, Regulación, Garantías y Limitaciones de los Derechos Fundamentales». Ius et Praxis, Vol. 11, No. 2: 15-64. Recuperado de http://dx.doi.org/10.4067/S0718-00122005000200002, el 4 de octubre de 2016.

11. ORGAZ, G. 2006. Cooperativas Agrarias. Régimen jurídico. Córdoba. La Cañada.

12. PAOLUCCI, L. 1999. Le societa cooperativa. Milano. Giuffre.

13. PIÑEIRO HARNECKER, C. 2014. "Diagnóstico preliminar de las cooperativas no agropecuarias en La Habana, Cuba». Recuperado de https:// www.ceec.uh.cu/file/569/download?token=VfqEKMZE, el 18 de abril de 2016.

14. RODRÍGUEZ MUSA, O. 2013. «La autonomía cooperativa y su expresión jurídica. Una aproximación crítica a su actual implementación legal en Cuba». Boletín de la Asociación Internacional de Derecho Cooperativo, No. 47: 129-155.

15. RODRÍGUEZ MUSA, O. 2017. La constitucionalización de la cooperativa. Una propuesta para su redimensionamiento en Cuba, Coletânea IBECOOP No. 1, Ed. Vincere Asociados, Brasilia-DF.

16. ROMERO, P. 2001. De los socios. Cooperativas. Comentarios a la Ley 27/1999, de 16 de julio. Madrid. Colegios Notariales de España.

17. SENENT VIDAL, M.J. 2011. «Estatutos sociales y otros documentos». En FAJARDO GARCÍA, G. (coordinadora): Cooperativas: Régimen Jurídico y Fiscal, Universitat de València, Generalitat Valenciana, Tirant lo Blanch (monografía 738), Valencia, 59-85.

18. TRISTÁ ARBEZÚ, G. 2015. "Cooperativas no Agropecuarias», conferencia ofrecida y reproducida a militantes del Partido Comunista de Cuba por la Comisión de Implementación de los Lineamientos del Partido y la Revolución.

19. VUOTTO, M. 2015. «Las cooperativas no agropecuarias y la transformación económica en cuba: políticas, procesos y estrategias», en Revista de Estudios Cooperativos (REVESCO), recuperado de http://dx.doi. org/10.5209/rev_REVE.2016.v120.49697, el 18 de abril de 2016. 


\section{Legislación}

1. Código de Comercio español de 1886 (anotado y actualizado). 1998. Ed. Félix Varela. La Habana.

2. Ley 95/2002, «Ley de Cooperativas de Producción Agropecuaria y de Créditos y Servicios». Gaceta Oficial Ordinaria No. 72 de 29 de noviembre de 2002.

3. Decreto-Ley 305/2012, "De las cooperativas no agropecuarias». Gaceta Oficial No. 053 Extraordinaria de 11 de diciembre de 2012.

4. Decreto-Ley 142/1993, «Sobre las Unidades Básicas de Producción Cooperativa». Gaceta Oficial No. 6 Extraordinaria de 21 de septiembre de 1993.

5. Decreto 309/2012, «Reglamento de las cooperativas no agropecuarias de primer grado». Gaceta Oficial No. 053 Extraordinaria de 11 de diciembre de 2012.

6. Acuerdo 5454 del 17 de mayo de 2005 del Comité Ejecutivo del Consejo de Ministros, «Reglamentos Generales de las Cooperativas de Producción Agropecuaria y Reglamento General de la Cooperativas de Créditos y Servicios». Gaceta Oficial No. 20 de 4 de julio de 2005.

7. Resolución 574/2012 del Ministerio de la Agricultura, «Reglamento General de las Unidades Básicas de Producción Cooperativa». Gaceta Oficial No. 037 Extraordinaria de 11 de septiembre de 2012.

8. Ley 116/2014, "Código de Trabajo». Gaceta Oficial No. 29 Extraordinaria de 17 de junio de 2014. 


\section{Derechos de autor}

El Boletín de la Asociación Internacional de Derecho Cooperativo es una revista de acceso abierto lo que significa que es de libre acceso en su integridad inmediatamente después de la publicación de cada número. Se permite su lectura, la búsqueda, descarga, distribución y reutilización legal en cualquier tipo de soporte sólo para fines no comerciales y según lo previsto por la ley; sin la previa autorización de la Editorial (Universidad de Deusto) o el autor, siempre que la obra original sea debidamente citada (número, año, páginas y DOI si procede) y cualquier cambio en el original esté claramente indicado.

\section{Copyright}

The International Association of Cooperative Law Journal is an Open Access journal which means that it is free for full and immediate access, reading, search, download, distribution, and lawful reuse in any medium only for non-commercial purposes, without prior permission from the Publisher or the author; provided the original work is properly cited and any changes to the original are clearly indicated. 